Case Report

\title{
Gastric Intussusceptions in a Red Corn Snake (Pantherophis guttatus) Associated with Cryptosporidiosis
}

\author{
Marjorie Bercier, ${ }^{1}$ Whitney Zoll, ${ }^{2}$ Justin F. Rosenberg, ${ }^{1}$ Robson Giglio, ${ }^{3}$ Lenice McCoy, ${ }^{3}$ \\ William L. Castleman, ${ }^{2}$ Matthew D. Johnson, ${ }^{1}$ and Darryl J. Heard ${ }^{1}$ \\ ${ }^{1}$ Department of Small Animal Clinical Sciences, College of Veterinary Medicine, University of Florida, \\ 2015 SW 16th Avenue, Gainesville, FL 32610, USA \\ ${ }^{2}$ Department of Infectious Diseases and Pathology, College of Veterinary Medicine, University of Florida, \\ 2015 SW 16th Avenue, Gainesville, FL 32610, USA \\ ${ }^{3}$ Department of Large Animal Clinical Sciences, College of Veterinary Medicine, University of Florida, \\ 2015 SW 16th Avenue, Gainesville, FL 32610, USA \\ Correspondence should be addressed to Marjorie Bercier; bercierm@ufl.edu
}

Received 7 February 2017; Accepted 18 April 2017; Published 7 May 2017

Academic Editor: Lysimachos G. Papazoglou

Copyright (C) 2017 Marjorie Bercier et al. This is an open access article distributed under the Creative Commons Attribution License, which permits unrestricted use, distribution, and reproduction in any medium, provided the original work is properly cited.

A 3-year-old female red corn snake (Pantherophis guttatus) was presented for a three-week history of anorexia and decreased defecations. On physical examination, a soft midbody intracoelomic swelling was palpated. Transcutaneous coelomic ultrasound revealed a target-like mass on a transverse section of the stomach, suggesting the presence of a gastrointestinal intussusception. On exploratory coeliotomy, a double compounded esophagogastric and gastroduodenal intussusception was diagnosed and reduced surgically. A gastropexy was also performed to prevent recurrence. On histopathology, the gastric glandular mucosa showed moderate to marked proliferation. Diffusely lining the luminal surface of glandular epithelium and free within the lumen were a myriad of protozoa consistent with Cryptosporidium sp. A diagnosis of chronic proliferative gastritis due to Cryptosporidium sp. was made based on these findings. Intussusceptions are rare in reptiles and are infrequently reported in snakes. This is the first report of a double compounded intussusception in a nonmammalian species and the first report of an intussusception involving the stomach in a snake with gastritis due to Cryptosporidium sp.

\section{Introduction}

Cryptosporidium spp. is an apicomplexan parasite that is known to affect mammals including humans, birds, fishes, amphibians, and reptiles $[1,2]$. Infections have been reported in more than 80 species of reptiles, including snakes, lizards, and tortoises [1]. Two main Cryptosporidium spp. are frequently reported in reptiles: Cryptosporidium serpentis and Cryptosporidium varanii. C. serpentis is highly pathogenic in snakes and causes chronic hypertrophic gastritis. Clinical signs include regurgitation, anorexia, muscle wasting, midbody swelling, and death $[2,3]$. The aim of this report is to describe the diagnosis and surgical treatment of a double compounded esophagogastric and gastroduodenal intussusception in a red corn snake (Pantherophis guttatus) with Cryptosporidium sp.-associated gastritis.

\section{Case Report}

An approximately 3-year-old, intact female, $260 \mathrm{~g}$ red corn snake (Pantherophis guttatus) was evaluated by the Zoological Medicine Service, University of Florida, for a midbody swelling and a three-week history of anorexia. The snake was purchased from a breeder 2.5 years priorly and did not have any previous health concerns. The reptile was kept in a tank with aspen bedding, in a room kept at $25^{\circ} \mathrm{C}$ throughout the year. During winter, supplemental heating was offered in one end of the tank using a heat lamp. Temperature and humidity in the enclosure were not monitored. The snake was fed a frozen-thawed adult mouse once weekly. During the feedings, the snake was transferred to a different container without any bedding, where it was left with the food item until consumed. The owner reported that the snake normally defecated 
regularly, but that for the past three to four weeks the stools had been dryer, smaller, and less frequent. The snake remained active at home and displayed a normal behaviour. The owner also had a wild caught, apparently healthy grey ratsnake (Pantherophis spiloides) kept in a separate tank.

During physical examination, the red corn snake was quiet, alert, and responsive, and appeared in good body condition. A soft, ventral, intracoelomic swelling $(6 \times 4 \mathrm{~cm})$ was palpable approximately at midbody and elicited discomfort to the patient when manipulated. Ultrasonography (Philips iU22 ultrasound machine, Philips Medical Systems, Bothell, WA 98021, USA), through multifrequency linear transducers, revealed a cylindrical structure lateral to the caudal liver, coursing caudally and medially to reside in proximity to the stomach. At this location, the segment thought to represent the stomach was markedly and focally fluid dilated. Caudally, from this region of dilation, an additional cylindrical segment surrounded those described previously, resulting in a concentric ring appearance (Figure 1). The central component of the concentric ring demonstrated blood flow, using color Doppler. Based on these findings, a gastrointestinal intussusception was suspected and surgery was recommended.

The snake was sedated with $0.1 \mathrm{mg} / \mathrm{kg}$ of body weight (BW) of hydromorphone (West-Ward, Eatontown, NJ 07724, USA) administered intramuscularly. Intubation with a $14 \mathrm{G}$ catheter was possible following the sedation and the animal was maintained on 1-2\% isoflurane (Piramal Healthcare Limited, Andhra Pradesh 502321, India) in a mixture of oxygen and nitrous oxide $(1 \mathrm{~L} / \mathrm{min}$ of each). The patient received positive intermittent manual ventilation at a rate of four breaths per minute. The patient was placed in left lateral recumbency, and the surgical site was prepped aseptically. A $10 \mathrm{~cm}$ incision was made two scale rows dorsal to the ventral scales. The incision followed along the edges of the scales, forming a scalloped pattern. The underlying muscle layers were incised at the end of the ribs. A Lonestar retractor (Jorgensen Labs Inc., Loveland, CO 80538, USA) was used to improve visualization of the coelomic cavity. After manipulation of the tissues, a gastrotomy was made laterally and spanned most of the length of the stomach and into the duodenum approximately $2 \mathrm{~cm}$. The esophagus was observed to be intussuscepted into the stomach (Figure 2(b)), and then this combination was further invaginated into the duodenum (Figure 2(a)) forming a second intussusception. A diagnosis of double compounded esophagogastric and gastroduodenal intussusception was made. The stomach was extracted from the duodenum and then the esophagus was extracted from the stomach. There were adhesions formed between the esophagus and stomach that were bluntly dissected in order to extract the esophagus from the stomach (Figure 2(c)). Grossly, the gastric longitudinal rugae appeared hypertrophied. Samples of gastric mucosa were placed into $10 \%$ buffered neutral formalin for histopathologic evaluation. The stomach was closed in two layers with 3-0 PDS (Ethicon LLC, Cincinnati, OH 45242, USA) with a Lembert pattern in the mucosa/submucosa layer, followed by a simple continuous pattern for the serosal muscularis layer. Due to the nature of the intussusception, the incision in the duodenum had a transverse as well as a longitudinal component. The transverse incision in the

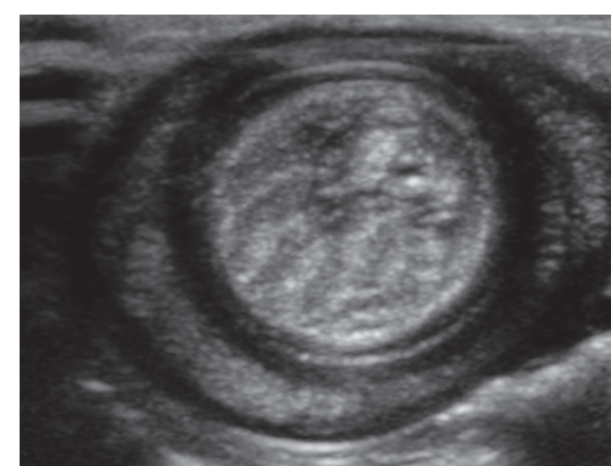

FIGURE 1: Short axis ultrasonographic image of the stomach demonstrating the typical target-like (multilayering) appearance seen in cases of intussusception.

duodenum was closed with four simple interrupted sutures using 4-0 PDS (Ethicon LLC, Cincinnati, OH 45242, USA). The longitudinal component was closed with a simple continuous pattern using 4-0 PDS. A gastropexy via interrupted circumcostal sutures was performed with 3-0 PDS to prevent recurrence of the intussusceptions. The body wall was closed routinely. The muscle layer was closed with 3-0 PDS in a simple continuous manner. The integument was closed with nine horizontal mattress and one simple interrupted sutures to cause an eversion of the scales using 3-0 PDS.

The patient was discharged the following day. Treatments included famotidine (Mylan Institutional LLC, Rockford, IL 661103 , USA) at $0.026 \mathrm{mg} / \mathrm{kg}$ BW SC q48h, ceftazidime (Hospira Worldwide Inc., Lake Forest, IL 60045, USA) at $22 \mathrm{mg} / \mathrm{kg}$ BW SC q72h, and meloxicam (Putney Inc., Portland, ME 04101, USA) at $0.1 \mathrm{mg} / \mathrm{kg}$ BW SC q48h. The snake was not fed for two weeks before reexamination. The owner provided a supplemental heat lamp in the tank to have a warm basking spot and a cooler temperate area. All bedding was removed from the enclosure and only clean newspaper or paper towels were used as a substrate.

In histologic sections of the stomach, epithelium on the gastric surface and in gastric glands was hyperplastic (Figure 3(a)). Dilation of the mucosal glands and fibrosis of the lamina propria were also evident. Myriad protozoa that were $2 \mu \mathrm{m}$ in diameter, eosinophilic to basophilic, and periodicacid-Schiff- (PAS-) positive and that had variably distinct $0.5-2 \mu \mathrm{m}$ basophilic nuclei were closely associated with the apical epithelial surfaces as well as being free within the lumen (Figure 3(b)). Small numbers of heterophils, lymphocytes, and plasma cells were in the lamina propria. A diagnosis of chronic proliferative gastritis due to Cryptosporidium sp. was made based on these findings.

Two weeks postoperatively, the snake was recovering well. The surgical site was clean, well apposed, and free of any discharge. The ventral surface associated with the incision was mildly distended and the scales appeared slightly dull. The owner had no concerns at that time, and the snake maintained BW. All medications were discontinued and the snake was tube-fed $2.5 \mathrm{~mL}$ of carnivore care (Oxbow Animal Health, Murdoch, NE 68407, USA) slurry to help encourage normal gastrointestinal movements and to administer a small first 


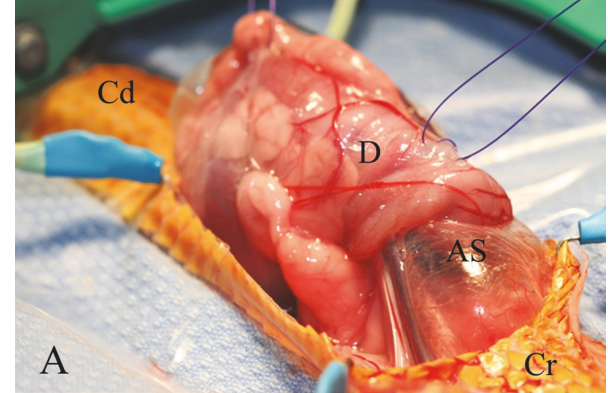

(a)

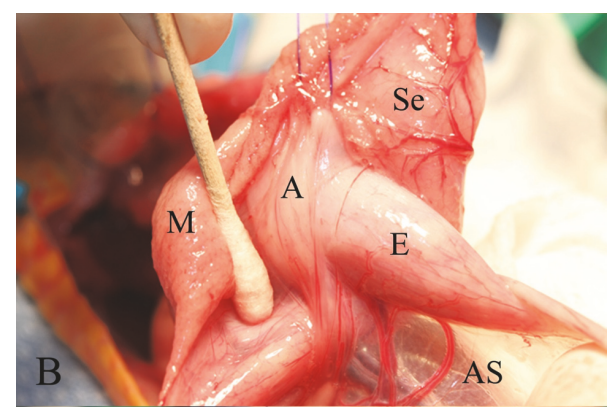

(b)

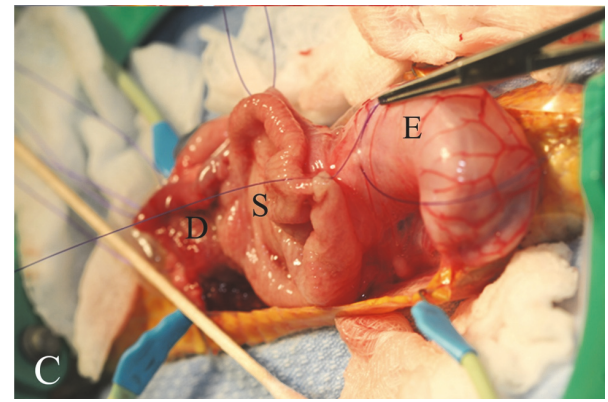

(c)

FIgure 2: (a) Gastroduodenal intussusception. The duodenum (D) and air sac (AS) are readily visible at the surgical site. The cranial (Cr) and caudal (Cd) directions of the snake are identified. (b) Esophagogastric intussusception. After the gastrotomy, adhesions (A) are identified between the esophagus (E) and the stomach's serosa (Se). The hypertrophied gastric mucosa (M) is visible. (c) Reduction of the double compounded intussusception. Depicted here are the esophagus (E), stomach (S), and duodenum (D) in their normal anatomic position.

meal. The owner was allowed to start refeeding the snake smaller prey items such as pinkies every five days for 1 month, and then increasing to one hopper every 5 days for another month, followed by adult mice once weekly as the preoperative husbandry practices.

At four weeks after surgery, the skin sutures were removed. A brief ultrasound exam was performed and did not show any obvious abnormalities around the stomach. The owner reported one abnormal bowel movement, described as diarrhea, that occurred four to five days after feeding the first pinkie mouse. The owner also reported that the snake had a normal shed two weeks priorly without any complications.

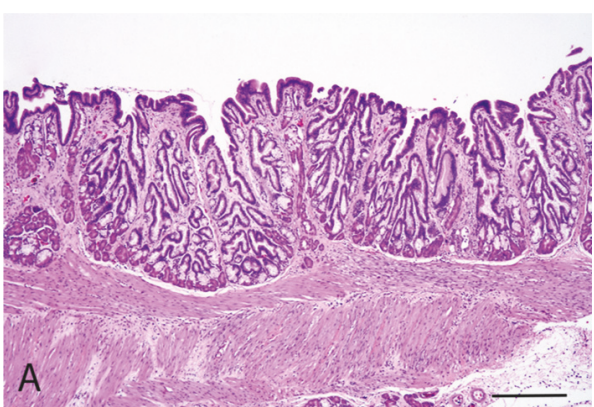

(a)

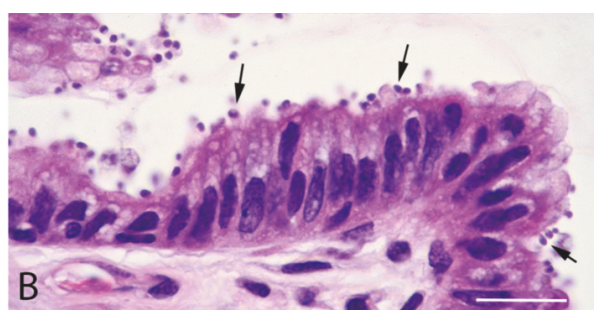

(b)

FIGURE 3: (a) Low power magnification image of chronically inflamed stomach with hyperplasia of surface and glandular epithelium. H\&E stain; bar $=142 \mu \mathrm{m}$. (b) High power magnification image of hyperplastic gastric glandular and surface epithelium with protozoa (arrows) closely associated with the apical surface membranes of the epithelial cells. Other protozoa are free in the lumen. $\mathrm{H} \& \mathrm{E}$, bar $=14 \mu \mathrm{m}$.

The animal had lost $30 \mathrm{~g}$ (approximately $12 \%$ of BW) since initial presentation, but this was expected due to the current feeding schedule. A gastric wash was performed and submitted for polymerase chain reaction (PCR) to speciate the Cryptosporidium present in the stomach. However, there were no organisms in the sample, and the PCR came back negative.

Unfortunately, at 15 months after surgery, the snake was found deceased in its enclosure. The owner reports that the snake would have intermittent episodes of regurgitation when it was fed adult mice but seemed to tolerate eating smaller food items without difficulties. The snake behaved normally, remained with a good appetite, and did not have other clinical signs until the day it was found dead. The cause of the regurgitations is unknown, but worsening of the chronic Cryptosporidium sp.-associated gastritis is suspected. The snake was not submitted for postmortem examination.

\section{Discussion}

An intussusception is an invagination of one segment (intussusceptum) of intestine into another (intussuscipiens). Intussuscepta are usually orad to the intussuscipiens (anterograde intussusception), but can also be aborad (retrograde intussusception). A retrospective study in domestic dogs and cats revealed that inflammatory disease and neoplasia were the most common causes of intestinal intussusceptions, respectively [4]. Intussusception has been infrequently described in reptiles [5]. It has been associated with monofilament fishing line ingestion in sea turtles $[6,7]$. In a report investigating 
gastrointestinal tract lesions in sea turtles, seven out of 136 animals had intestinal intussusceptions, sometimes leading to necrosis of the intestinal mucosa [7]. An ileocolic intussusception has been reported in a pine snake (Pituophis melanoleucus), who died shortly after a permanent ileostomy was performed [8]. Gastric intussusceptions have not previously been reported in reptiles. The Cryptosporidium sp.associated gastritis likely contributed to the development of this snake's intussusceptions, in a similar fashion to what inflammatory diseases do in dogs.

In domestic dogs, transcutaneous abdominal ultrasonography is a highly sensitive (100\%), specific (97.8\%), and accurate $(98.4 \%)$ method of diagnosis of intestinal intussusception, characterized as a target-like mass visible on transverse sections [9]. Due to their unique anatomy, characterized by their limbless, tubular, and elongated bodies, intussusception appears to be readily palpable in snakes. However, such finding is not specific and other differentials should be ruled out. Transcutaneous coelomic ultrasonography was a useful confirmatory diagnostic tool in this case, revealing the typical concentric rings in the transverse section of the stomach.

There is discrepancy in the literature regarding the nomenclature of complex intussusceptions. As described here, a double compounded intussusception is a combination of two anterograde intussusceptions at a given location [10]. A double intussusception (DI) involves two distinct intussusceptions at separate points of the bowel [10]. In the veterinary literature, the term DI has been used interchangeably for both entities. DI has been described in dogs [11-15] and cats [16], mostly of young age.

Two main Cryptosporidium spp. are frequently reported in reptiles. C. serpentis is a common pathogen of snakes and is known to cause hypertrophic gastritis. C. varanii (previously known as Cryptosporidium saurophilum) is most commonly reported in lizards; however it has also been seen in snakes [17-19]. Cryptosporidium sp.-associated enteritis without evidence of gastritis has been reported in snakes, but the species of the causative agent was not determined [20]. Coinfection with an adenovirus likely increased one snake's susceptibility to cryptosporidiosis by creating immunodeficiency [21]. Other factors facilitating susceptibility of snakes to Cryptosporidium sp. gastritis are believed to be stress of captivity, translocations, and reproduction [21].

Clinical signs of $C$. serpentis gastritis include regurgitation, anorexia, muscle wasting, midbody swelling, and death $[2,3]$. Diagnosis of $C$. serpentis in snakes can be made by acidfast staining or PCR on fecal samples, gastric lavage samples, regurgitated material, or gastric biopsies $[3,19]$. Gross appearance of the gastric mucosa of affected snakes includes focal or diffuse hyperemia, cobblestoned appearance, thickened mucosa, lack of rugal folds, accentuation of longitudinal ridges, and edema $[2,3,21]$. These changes can result in apparent narrowing of gastric lumen. On histopathology, chronic gastritis is characterized by proliferation, increased tortuosity and mucoid metaplasia of the pyloric mucosal glands, fibrosis around the glands, hyperplasia of mucosal lining cells, and infiltration of heterophils and lymphocytes in the lamina propria [3]. Intralesional and intraluminal Cryptosporidium sp. are often visible $[2,3,21]$. There is no known effective treatment for Cryptosporidium sp. infections in snakes. Savanna monitors have been successfully treated for cryptosporidiosis with gastric administration of hyperimmune bovine colostrum, but this treatment only decreased the parasite load and did not completely clear infection in snakes and leopard geckos [22-24]. For this reason, no treatment was implemented for cryptosporidiosis in this case.

This is the first report of a double compounded intussusception in a reptile, and the first report of an intussusception involving the stomach in a snake with gastritis due to Cryptosporidium sp. Transcutaneous coelomic ultrasonography was helpful in the diagnosis of the condition. Surgical reduction of the intussusceptions and gastropexy resulted in successful resolution of all clinical signs, with the exception of intermittent regurgitations when fed a large food item. The snake lived up to 15 months after surgery, and although no postmortem examination was performed, Cryptosporidium sp.-associated chronic proliferative gastritis is considered the most likely cause of death.

\section{Conflicts of Interest}

The authors declare that there are no conflicts of interest regarding the publication of this paper.

\section{References}

[1] R. Fayer, "Taxonomy and species delimitation in Cryptosporidium," Experimental Parasitology, vol. 124, no. 1, pp. 90-97, 2010.

[2] P. J. O’Donoghue, "Cryptosporidium and cryptosporidiosis in man and animals," International Journal for Parasitology, vol. 25, no. 2, pp. 139-195, 1995.

[3] S. N. S. Cerveny, M. M. Garner, J. J. D’Agostino, S. R. Sekscienski, M. E. Payton, and M. R. Davis, "Evaluation of gastroscopic biopsy for diagnosis of Cryptosporidium sp. Infection in snakes," Journal of Zoo and Wildlife Medicine, vol. 43, no. 4, pp. 864-871, 2012.

[4] A. S. Levien and S. J. Baines, "Histological examination of the intestine from dogs and cats with intussusception," Journal of Small Animal Practice, vol. 52, no. 11, pp. 599-606, 2011.

[5] M. M. Garner, "Overview of biopsy and necropsy techniques," in Reptile Medicine and Surgery, D. R. Mader, Ed., pp. 569-580, Saunders, St. Louis, Mo, USA, 2nd edition, 2006.

[6] A. Di Bello, C. Valastro, D. Freggi, O. R. Lai, G. Crescenzo, and D. Franchini, "Surgical treatment of injuries caused by fishing gear in the intracoelomic digestive tract of sea turtles," Diseases of Aquatic Organisms, vol. 106, no. 2, pp. 93-102, 2013.

[7] J. Orós, P. Calabuig, and S. Déniz, "Digestive pathology of sea turtles stranded in the Canary Islands between 1993 and 2001," Veterinary Record, vol. 155, no. 6, pp. 169-174, 2004.

[8] M. A. Wosar and G. A. Lewbart, "Ileocolic intussusception in a pine snake (Pituophis melanoleucus)," Veterinary Record, vol. 158, no. 20, pp. 698-699, 2006.

[9] M. N. Patsikas, S. Jakovljevic, N. Moustardas, L. G. Papazoglu, G. M. Kazakos, and A. K. Dessiris, "Ultrasonographic signs of intestinal intussusception associated with acute enteritis or gastroenteritis in 19 young dogs," Journal of the American Animal Hospital Association, vol. 39, no. 1, pp. 57-66, 2003.

[10] F. Destro, N. Cantone, M. Maffi, T. Gargano, and M. Lima, "An interesting case of double compound intussusception without 
intestinal occlusion in a 5-year-old boy," European Journal of Pediatric Surgery Reports, vol. 2, no. 1, pp. 20-22, 2014.

[11] M. Atray, M. Raghunath, T. Singh, and N. S. Saini, "Ultrasonographic diagnosis and surgical management of double intestinal in tussusception in 3 dogs," Canadian Veterinary Journal, vol. 53, no. 8, pp. 860-864, 2012.

[12] E. Bellezza, E. Bianchini, S. Pettinelli, G. Angeli, and L. Leonardi, "Intestinal plasmacytoma causing colocolic double intussusception in an adult dog," Journal of Small Animal Practice, vol. 57, no. 12, pp. 718-718, 2016.

[13] C. L. Joy and J. M. Patterson, "Short bowel syndrome following surgical correction of a double intussusception in a dog," Canadian Veterinary Journal, vol. 19, no. 9, pp. 254-259, 1978.

[14] J. M. Kim, S. S. Kang, G. Kim, and S. H. Choi, "Double intussusception in a Shih-Tzu puppy: the triple-circle sign," Pakistan Veterinary Journal, vol. 32, no. 2, pp. 292-294, 2012.

[15] P. A. Okewole, P. S. Odeyemi, T. Cole, A. E. Itodo, and M. Odugbo, "Double intussusception fatally complicated by clostridial infection in a dog (a case report)," British Veterinary Journal, vol. 145, no. 3, pp. 291-292, 1989.

[16] C. R. Bellenger and J. A. Beck, "Intussusception in 12 cats," Journal of Small Animal Practice, vol. 35, no. 6, pp. 295-298, 1994.

[17] C. Deming, E. Greiner, and E. W. Uhl, "Prevalence of cryptosporidium infection and characteristics of oocyst shedding in a breeding colony of leopard geckos (Eublepharis macularius)," Journal of Zoo and Wildlife Medicine, vol. 39, no. 4, pp. 600-607, 2008.

[18] J. Plutzer and P. Karanis, "Molecular identification of a Cryptosporidium saurophilum from corn snake (Elaphe guttata guttata)," Parasitology Research, vol. 101, no. 4, pp. 1141-1145, 2007.

[19] B. Richter, N. Nedorost, A. Maderner, and H. Weissenböck, "Detection of Cryptosporidium species in feces or gastric contents from snakes and lizards as determined by polymerase chain reaction analysis and partial sequencing of the $18 \mathrm{~S}$ ribosomal RNA gene," Journal of Veterinary Diagnostic Investigation, vol. 23, no. 3, pp. 430-435, 2011.

[20] A. I. Brower and M. R. Cranfield, "Cryptosporidium sp.-associated enteritis without gastritis in rough green snakes (Opheodrys aestivus) and a common garter snake (Thamnophis sirtalis)," Journal of Zoo and Wildlife Medicine, vol. 32, no. 1, pp. 101-105, 2001.

[21] D. Mahapatra, M. Reinhard, and H. K. Naikare, "Adenovirus and cryptosporidium co-infection in a Corn Snake (elaphae guttata guttata)," Journal of Zoo and Wildlife Medicine, vol. 44, no. 1, pp. 220-224, 2013.

[22] T. K. Graczyk, M. R. Cranfield, P. Helmer, R. Fayer, and E. F. Bostwick, "Therapeutic efficacy of hyperimmune bovine colostrum treatment against clinical and subclinical Cryptosporidium serpentis infections in captive snakes," Veterinary Parasitology, vol. 74, no. 2-4, pp. 123-132, 1998.

[23] T. K. Graczyk, M. R. Cranfield, and E. F. Bostwick, "Hyperimmune bovine colostrum treatment of moribund leopard geckos (Eublepharis macularius) infected with Cryptosporidium sp.," Veterinary Research, vol. 30, no. 4, pp. 377-382, 1999.

[24] T. K. Graczyk, M. R. Cranfield, and E. F. Bostwick, "Successful hyperimmune bovine colostrum treatment of savanna monitors (Varanus exanthematicus) infected with Cryptosporidium sp.," Journal of Parasitology, vol. 86, no. 3, pp. 631-632, 2000. 

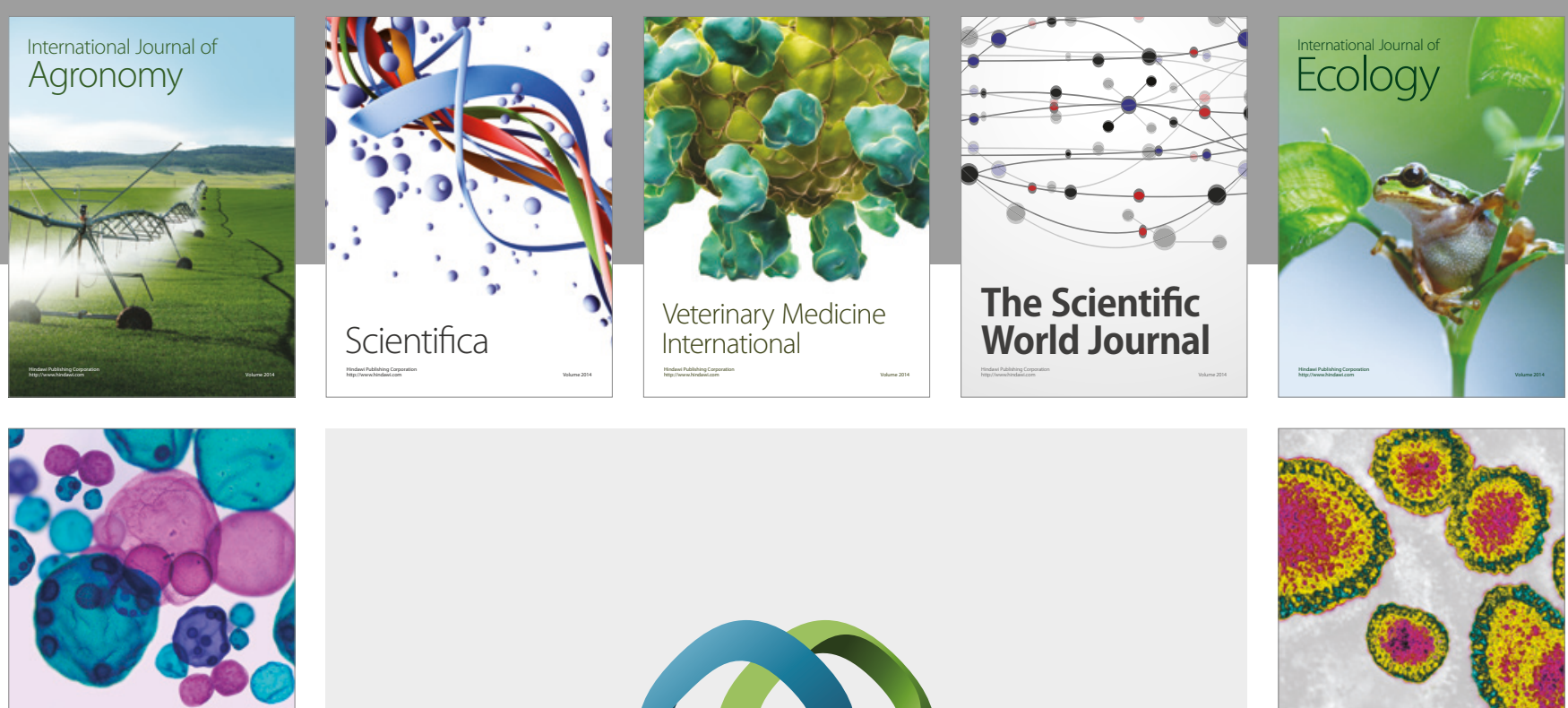

\section{International Journal of} Microbiology
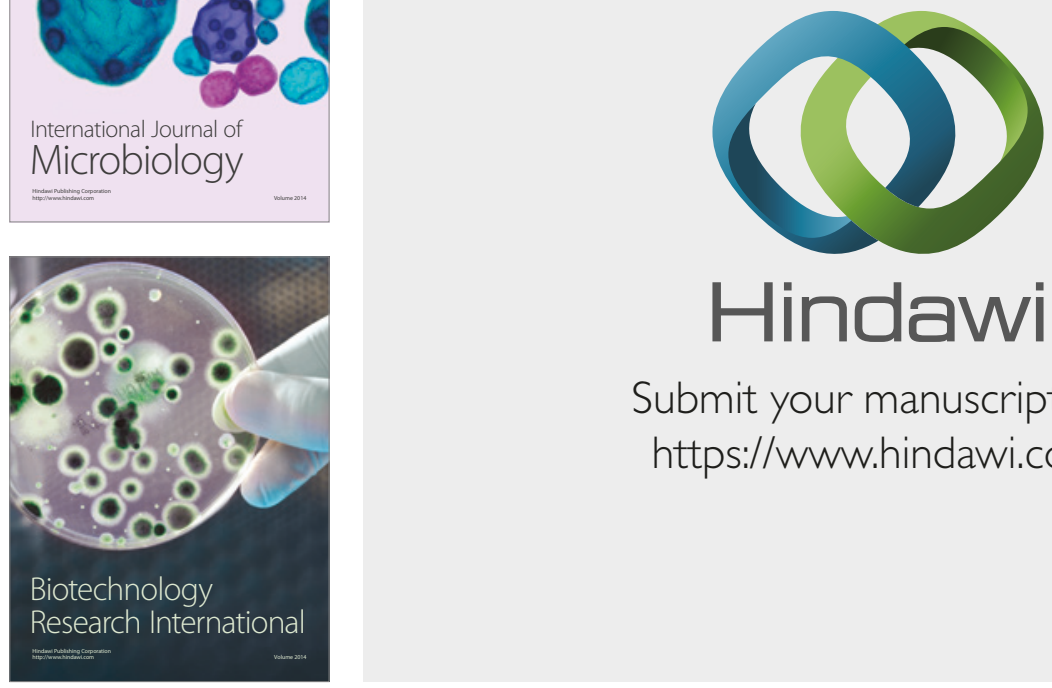

Submit your manuscripts at

https://www.hindawi.com
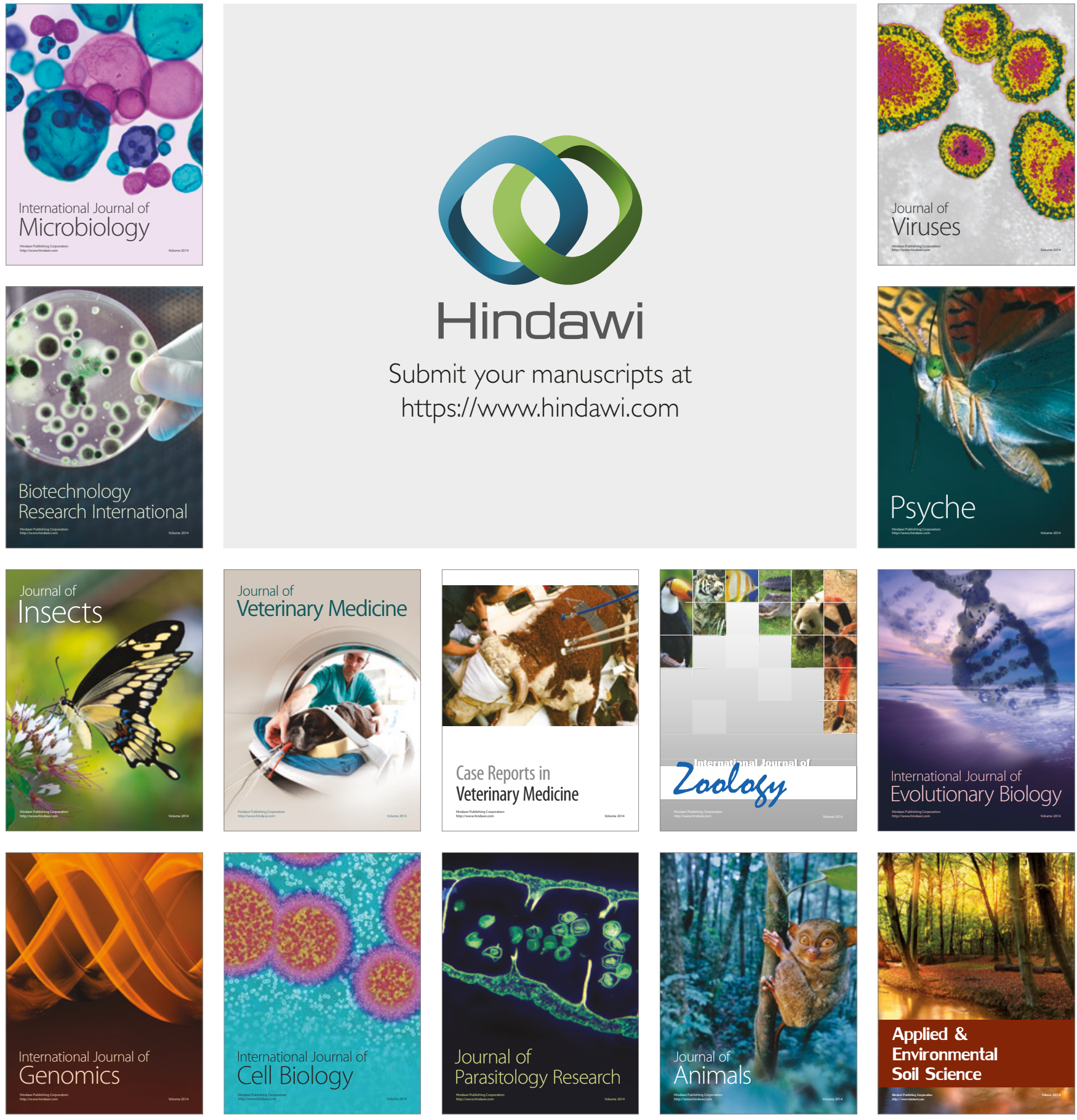